\title{
Intoxicação por medicamentos: uma revisão de literatura com abordagem no tratamento
}

\author{
Drug poisoning: a literature review with a treatment approach
}

Intoxicación por drogas: una revisión de la literatura con un enfoque de tratamiento

Victória Toledo Silva ${ }^{1}$, Letícia Maria Machado Coelho , Dayana Bomfim Santos ${ }^{1}$, Larissa Silva Martins $^{1}$, Gérsika Bitencourt Santos ${ }^{1 *}$.

\begin{abstract}
RESUMO
Objetivo: Analisar estudos sobre intoxicações medicamentosas, de modo a reunir os principais tratamentos e antídotos utilizados, visando contribuir para rumos de futuras investigações. Métodos: Trata-se de uma revisão de literatura, cujos artigos foram selecionados nas bases de dados PubMED, SciELO e LILACS, publicados a partir do ano de 2015, nos idiomas inglês, espanhol ou português. Resultados: Foram encontrados 2092 artigos ao todo. Após aplicação dos critérios de exclusão e de inclusão, obteve-se 17 artigos para comporem a amostra. Dentre os estudos selecionados, a intoxicação por paracetamol foi a mais relatada. $O$ suporte vital ao paciente deve ser priorizado. $O$ carvão ativado, a lavagem gástrica e a alcalinização da urina foram as formas de tratamento mais encontradas, sendo a hemodiálise, a hemoperfusão e a infusão de lipídios alternativas terapêuticas. Além disso, listou-se os principais antídotos conhecidos para algumas intoxicações específicas. Considerações Finais: As intoxicações por medicamentos constituem um problema de âmbito mundial, sendo necessárias políticas de prevenção, a fim de evitar o crescimento da automedicação.
\end{abstract}

Palavras-chave: Intoxicação, Medicamentos, Tratamento, Uso abusivo de medicamentos.

\begin{abstract}
Objective: To analyze studies on drug intoxication in order to gather the main treatments and antidotes used, aiming to contribute to future research directions. Methods: This is a literature review, whose articles were selected from the PubMED, SciELO and LILACS databases, published from the year 2015, in English, Spanish or Portuguese. Results: a total of 2092 articles were found. After applying the exclusion and inclusion criteria, we obtained 17 articles to compose the sample. Among the selected studies, paracetamol intoxication was most reported. Vital support to the patient should be prioritized. Activated charcoal, gastric lavage, and urine alkalinization were the most found forms of treatment, with hemodialysis, hemoperfusion, and lipid infusion being alternative therapies. In addition, the main known antidotes for some specific intoxications were listed. Final considerations: Drug intoxication is a worldwide problem, and prevention policies are necessary in order to avoid the growth of the self-medication.
\end{abstract}

Key words: Poisoning, Drugs, Treatment, Abuse of drugs.

\section{RESUMEN}

Objetivo: Analizar los estudios sobre intoxicaciones medicamentosas, de manera que se reúnan los principales tratamientos y antídotos utilizados, con vistas a contribuir a los rumores de futuras investigaciones. Métodos: Se trata de una revisión bibliográfica, cuyos artículos fueron seleccionados de las bases de datos

1 Universidade José do Rosário Vellano (UNIFENAS), Alfenas - MG. *E-mail: gersika.bitencourt@unifenas.br SUBMETIDO EM: 2/2021

PUBLICADO EM: 3/2021 
PubMED, SciELO y LILACS, publicados a partir del año 2015, en inglés, español o portugués. Resultados: se encontró un total de 2092 artículos. Tras aplicar los criterios de exclusión e inclusión, se obtuvieron 17 artículos para componer la muestra. Entre los estudios seleccionados, la intoxicación por paracetamol fue la más relacionada. El apoyo vital al paciente debe ser prioritario. El carbón activado, el lavado gástrico y la alcalinización de la orina fueron las formas de tratamiento más frecuentes, siendo la hemodiálisis, la hemoperfusión y la infusión de lípidos las terapias alternativas. Además, se enumeran los principales antídotos conocidos para algunas intoxicaciones específicas. Consideraciones finales: Las intoxicaciones por drogas son un problema mundial, y se necesitan políticas de prevención para evitar el crecimiento de la automedicación.

Palabras clave: Intoxicación, Medicamentos, Tratamiento, Abuso de medicamentos.

\section{INTRODUÇÃO}

Os medicamentos são essenciais para a capacidade resolutiva dos serviços de saúde por estarem associados a profilaxia, cura, paliação ou diagnóstico. Entretanto, a utilização de medicamentos favorece o surgimento de problemas relacionados aos mesmos. As intoxicações medicamentosas causam inúmeras mortes, sendo por esse motivo consideradas um problema de saúde pública. As principais causas das mortes são resultadas de uso abusivo, relacionado à automedicação ou ao autoextermínio, ou uso acidental (OLIVEIRA JFM, et al., 2017).

De acordo com o Sistema Nacional de Informações Toxicológicas (SINITOX), que é responsável por divulgar estatísticas brasileiras anuais referentes a casos novos de intoxicações registrados pelos Centros de Assistência e Informação Toxicológica (CEATOX), os fármacos continuam sendo os principais agentes tóxicos responsáveis por casos de intoxicações em humanos desde o ano de 1994. As intoxicações são ocasionadas por substâncias endógenas e exógenas, as quais são capazes de alterar a fisiologia do organismo e proporcionar alterações bioquímicas (THORNE PS, 2013).

As intoxicações medicamentosas constituem-se por diversos sintomas causados pelo medicamento ingerido, inalado, injetado ou de uso tópico, em doses superiores ao uso terapêutico. Cada droga apresenta suas peculiaridades em um quadro de sinais e sintomas diversos, com características específicas. Dentre as causas para tal problemática, tem-se a falta de regularização de publicidades e a falta de programas educativos, a facilidade de compra de fármacos sem receita médica e o uso indiscriminado de medicamentos (GONÇALVES CA, et al., 2017).

Para cada tipo de intoxicação existem normas e protocolos específicos de tratamento, sendo necessárias algumas condutas para o socorro imediato às vítimas. Nas intoxicações agudas, a avaliação clínica e o tratamento inicial devem ser o primeiro passo para a identificação e correção de situações de risco. A identificação do medicamento e a determinação da sua concentração plasmática inicial são informações valiosas para o tratamento adequado, quando é possível obtê-las. Em alguns casos, não é possível determinar o agente tóxico envolvido no episódio e a identificação de sinais e sintomas através do reconhecimento das síndromes neurotóxicas permite acompanhar o quadro clínico do paciente, para seguimento do tratamento e considerações especiais cabíveis. É através do reconhecimento da síndrome que se tenta orientar os testes diagnósticos e o tratamento (NÓBREGA HOS, 2015).

O presente artigo tem como objetivo apresentar uma revisão de literatura sobre o tema Intoxicações por Medicamentos, com abordagem no tratamento, visando o esclarecimento sobre o assunto, de forma a contribuir para o meio científico.

\section{MÉTODOS}

Para esta revisão de literatura, utilizou-se a estratégia PICo, descrita por Cardoso V (2019), cujos acrômios são "P": população; "l": interesse; "Co": contexto. A pergunta científica levantada por meio da estratégia foi a seguinte: quais os tratamentos mais utilizados em intoxicações medicamentosas em humanos? A população 
em questão avaliada foi de pessoas intoxicadas por medicamentos, com interesse em intoxicações agudas e crônicas, cujo contexto era o de tratamento e suporte hospitalar.

Utilizou-se as bases de dados National Library of Medicine (PubMED), Scientific Electronic Library Online (SciELo) e Literatura Latino-Americana em Ciências da Saúde (LILACS), buscando-se os descritores de forma isolada ou combinada com o operador booleano "AND". Na plataforma PubMED, buscou-se pelos descritores combinados "Treatment" AND "Antidote" AND "Drug Intoxication". Na LILACS, utilizou-se os descritores "Treatment of Drug Poisoning" e "Intoxicação" AND "Medicamento" AND "Tratamento" AND "Antídoto". Na SciELo, utilizou-se "Treatment of Drug Intoxication" e "Intoxicação por Fármacos". Como critério de inclusão, adotou-se: idioma inglês, espanhol ou português, com data de publicação posterior ao ano de 2015, disponibilizados na íntegra. Foram excluídos artigos duplicados, sem consistência na abordagem ou que fugiram ao tema proposto pela pergunta científica norteadora. A estratégia de busca está descrita na tabela a seguir (Tabela 1).

Tabela 1 - Distribuição dos estudos obtidos através da busca pelos descritores, nas bases de dados SciELO, PubMED e LILACS.

\begin{tabular}{cccccc}
\hline $\begin{array}{c}\text { Bases de } \\
\text { Dados }\end{array}$ & Descritores & $\begin{array}{c}\text { No de } \\
\text { estudos } \\
\text { encontrados }\end{array}$ & $\begin{array}{c}\text { No de } \\
\text { estudos } \\
\text { selecionados } \\
\text { pelo ano } \\
(\geq 2015)\end{array}$ & $\begin{array}{c}\text { No de estudos } \\
\text { selecionados } \\
\text { para análise }\end{array}$ & $\begin{array}{c}\text { No de } \\
\text { estudos } \\
\text { selecionados } \\
\text { como } \\
\text { amostra }\end{array}$ \\
\hline Lilacs & $\begin{array}{c}\text { "Treatment of } \\
\text { drug poisoning" }\end{array}$ & 224 & 58 & 7 & 5 \\
\hline $\begin{array}{c}\text { "Intoxicação AND } \\
\text { Medicamento } \\
\text { AND Tratamento } \\
\text { AND Antídoto" }\end{array}$ & 515 & 98 & 5 & 3 \\
\hline $\begin{array}{c}\text { "Treatment of } \\
\text { drug intoxication" }\end{array}$ & 37 & 11 & 7 & 3 \\
\hline $\begin{array}{c}\text { "Intoxicação por } \\
\text { fármacos" }\end{array}$ & 10 & 6 & 1 & 1 \\
\hline PubMED & $\begin{array}{c}\text { "Treatment AND } \\
\text { Antidote AND } \\
\text { Drug intoxication" }\end{array}$ & 1306 & 264 & 22 & 5 \\
\hline
\end{tabular}

Fonte: Silva VT, et al., 2021.

\section{RESULTADOS}

Após a busca pelos descritores nas bases de dados, foram identificados, ao todo, 2092 artigos. Após aplicar os critérios de exclusão e de inclusão, obteve-se uma amostra para esta revisão de 17 artigos. Os estudos englobam 5 relatos de caso, 11 revisões de literatura e 1 estudo analítico.

Os artigos selecionados como amostra foram agrupados de acordo com os autores, ano, meio de publicação, país de origem e os principais achados (Quadro 1). Quanto à distribuição dos estudos, observase que 6 foram publicados no ano de 2018, 4 no ano de 2015, 2 nos anos de 2016, 2017 e 2019 e 1 no ano de 2020. A origem das publicações é variada, sendo que a maioria é latino-americana (7), seguida de 5 publicações norte-americanas, 3 europeias, 1 canadense e 1 australiana, evidenciando que a problemática de intoxicação medicamentosa é de escala mundial. 
Quadro 1 - Síntese dos autores, ano, revista, país e principais achados sobre intoxicação por medicamentos.

\begin{tabular}{|c|c|c|c|}
\hline $\mathbf{N}$ & Autores (Ano) & Revista-País & Principais achados \\
\hline 1 & $\begin{array}{l}\text { CASTILHO MM, } \\
\text { et al. (2018) }\end{array}$ & $\begin{array}{l}\text { Revista Colombiana } \\
\text { de Nefrologia - } \\
\text { Colômbia }\end{array}$ & $\begin{array}{l}\text { As principais condutas para o tratamento de intoxicações agudas por lítio é o cumprimento do protocolo } \\
\text { ABC e cuidados de suporte, com hidratação com soro fisiológico }(0,9 \%) \text {. Pode-se recorrer ao tratamento } \\
\text { com carvão ativado via oral ou kayexelate, apesar destes não serem amplamente recomendados. A } \\
\text { hemodiálise se mostra uma opção de tratamento quando outras terapias falham. }\end{array}$ \\
\hline 2 & $\begin{array}{c}\text { SEPULVEDA } \\
\text { RA, et al. (2018) }\end{array}$ & $\begin{array}{c}\text { Revista Médica do Chile } \\
\text { - Chile }\end{array}$ & $\begin{array}{l}\text { O tratamento se dá pelo suporte básico à vida, podendo ser realizada a lavagem gástrica, o uso de carvão } \\
\text { ativado ou a alcalinização da urina, a fim de favorecer a eliminação renal. A hemodiálise pode ser } \\
\text { necessária em casos mais graves. }\end{array}$ \\
\hline 3 & $\begin{array}{l}\text { GARZÓN RGC, } \\
\text { et al. (2018) }\end{array}$ & $\begin{array}{l}\text { Universitas Medica- } \\
\text { Colômbia }\end{array}$ & $\begin{array}{l}\text { O tratamento recomendado envolve a acetilcisteína, antídoto de primeira escolha. Outro medicamento } \\
\text { utilizado é a metionina. Alguns fármacos têm sido estudados para reverter a intoxicação por paracetamol, } \\
\text { tais como a metformina e acetilcarnitina, além das vitaminas } \mathrm{C} \text { e E. }\end{array}$ \\
\hline 4 & $\begin{array}{l}\text { PÉREZ MM, et } \\
\text { al. (2019) }\end{array}$ & $\begin{array}{l}\text { Horizonte de Saúde - } \\
\text { Cuba }\end{array}$ & $\begin{array}{l}\text { O tratamento para intoxicações por barbitúricos se dá, primeiramente, pelo suporte vital, com controle dos } \\
\text { sistemas cardiovascular, respiratório e neurológico. Deve-se prevenir uma hipoglicemia com } \\
\text { hiperglicemiantes. As principais formas de descontaminação são lavagem gástrica e carvão ativado. A } \\
\text { alcalinização da urina é indicada para aumentar a excreção renal do fármaco. Em casos mais graves, } \\
\text { indica-se a hemodiálise ou hemoperfusão. }\end{array}$ \\
\hline 5 & $\begin{array}{l}\text { SKOLNIK A e } \\
\text { MONAS J. } \\
\quad(2017)\end{array}$ & $\begin{array}{l}\text { Emerg Med Clin North } \\
\text { Am - Estados Unidos }\end{array}$ & $\begin{array}{l}\text { A naloxona é um antídoto comum em intoxicações por opioides, os quais causam depressão respiratória. } \\
\text { Intoxicações por medicamentos cardíacos antagonistas de canais de cálcio e beta adrenérgicos, } \\
\text { causadores de bradicardia, podem ser tratados com atropina. O bloqueio de canais de sódio em } \\
\text { intoxicações por antidepressivos tricíclicos e antipsicóticos são causadoras de taquicardia, e tem como } \\
\text { tratamento o bicarbonato de sódio. }\end{array}$ \\
\hline 6 & $\begin{array}{l}\text { CHIEW AL, et } \\
\text { al. (2020) }\end{array}$ & $\begin{array}{l}\text { Medical Journal of } \\
\text { Australia -Austrália }\end{array}$ & $\begin{array}{l}\text { Em intoxicações agudas com altas doses de paracetamol ingeridas em até } 2 \text { horas, deve-se administrar } \\
\text { carvão ativado, que também é administrado em doses menores que } 30 \mathrm{~g} \text {, com menos de } 4 \text { horas de } \\
\text { ingestão. Pacientes que apresentam risco de hepatotoxicidade devem receber acetilcisteína intravenosa. }\end{array}$ \\
\hline 7 & $\begin{array}{l}\text { TUÑÓN JGP, et } \\
\text { al. (2020) }\end{array}$ & $\begin{array}{l}\text { Critical Medicine } \\
\text { (Mexican College of } \\
\text { Critical Medicine) - } \\
\quad \text { México }\end{array}$ & $\begin{array}{l}\text { As medidas de suporte vital devem ser priorizadas no tratamento de intoxicação por carbamazepina, além } \\
\text { de evitar a absorção gástrica por meio da lavagem e da administração de carvão ativado em doses } \\
\text { múltiplas. A cimetidina pode contribuir para o tratamento. Quando tais medidas não se fazem suficientes, } \\
\text { recorre-se à hemodiálise ou hemoperfusão. }\end{array}$ \\
\hline
\end{tabular}




\begin{tabular}{|c|c|c|c|}
\hline $\mathbf{N}$ & Autores (Ano) & Revista-País & Principais achados \\
\hline 8 & $\begin{array}{c}\text { MARTINS LS e } \\
\text { FILHO, AA } \\
(2018)\end{array}$ & $\begin{array}{l}\text { Revista Médica de } \\
\text { Minas Gerais - Brasil }\end{array}$ & $\begin{array}{l}\text { O tratamento para intoxicações por betabloqueadores deve ser iniciado pelo suporte básico à vida, } \\
\text { mantendo as vias aéreas livres e a circulação sanguínea. A lavagem gástrica é escolha indicada para até } \\
2 \text { horas de ingestão. Para evitar a absorção, usa-se carvão ativado. O glucagon é utilizado em } \\
\text { intoxicações por betabloqueadores, pois trata a bradicardia e a hipotensão. A insulina também é utilizada, } \\
\text { melhorando o desempenho cardíaco. A emulsão lipídica é um tratamento alternativo para tratar } \\
\text { intoxicação por drogas lipofílicas, podendo ser utilizada em quadros de instabilidade hemodinâmica } \\
\text { graves. }\end{array}$ \\
\hline 10 & $\begin{array}{l}\text { DIAZ M, et al. } \\
\qquad(2015)\end{array}$ & $\begin{array}{c}\text { Ato Toxicológico } \\
\text { Argentino - Argentina }\end{array}$ & $\begin{array}{l}\text { A isoniazida é um medicamento para tratamento de tuberculose. O tratamento deve ser iniciado com o } \\
\text { suporte à vida. Medidas que evitem a absorção do medicamento devem ser consideradas, tais como a } \\
\text { lavagem gástrica, o uso de carvão ativado, uso de leite de magnésia ou sorbitol } 70 \% \text {. O antídoto específico } \\
\text { é a vitamina B6 (piridoxina), de modo a diminuir quadros convulsivos e corrigir a acidose metabólica. Os } \\
\text { benzodiazepínicos podem ser outra opção de escolha anticonvulsivante. }\end{array}$ \\
\hline 12 & $\begin{array}{l}\text { BARTLETT D } \\
(2017)\end{array}$ & $\begin{array}{l}\text { Critical Care Nurse - } \\
\text { Estados Unidos }\end{array}$ & $\begin{array}{l}\text { Para tratamento de intoxicações causadoras da síndrome da serotonina, tem-se primeiramente o suporte } \\
\text { à vida e o tratamento dos sintomas, como a substituição de fluídos e o uso de benzodiazepínicos, a fim } \\
\text { de diminuir a pressão arterial, a taquicardia e a temperatura. As terapias com antídotos ainda estão sendo } \\
\text { avaliadas. A ciproheptadina é um anti-histamínico utilizado em casos de síndrome serotoninérgica, pois } \\
\text { tem propriedades antagonistas aos receptores serotoninérgicos. O antipsicótico olanzapine também } \\
\text { aparenta sucesso para reverter os sintomas da síndrome. A infusão de lipídeos se mostrou benéfica para } \\
\text { diminuir hiperreflexia e rigidez. Em casos graves, há casos em que a dexmetedomidina, um } \\
\text { adrenoreceptor agonista, melhorou a clínica do paciente. Tratamento com clorpromazina, propranolol } \\
\text { bromocriptina e dantroleno se mostraram sem sucesso. }\end{array}$ \\
\hline
\end{tabular}




\begin{tabular}{|c|c|c|c|}
\hline $\mathbf{N}$ & Autores (Ano) & Revista-País & Principais achados \\
\hline 13 & $\begin{array}{l}\text { PERNNIGA E, } \\
\text { et al. (2015) }\end{array}$ & $\begin{array}{l}\text { Basic \& Clinical Phar- } \\
\text { macology \& Toxicology } \\
\text { - Estados Unidos }\end{array}$ & $\begin{array}{l}\text { A sobredosagem de benzodiazepínicos tem como clínica o rebaixamento de consciência. Quando } \\
\text { relacionados com o álcool, tem a piora no quadro. Para prevenir a depressão do sistema respiratório e } \\
\text { diminuir o efeito sedativo, utiliza-se seu antídoto, o flumazenil, que bloqueia os receptores } \\
\text { benzodiazepínicos. O tratamento tem alguns efeitos adversos, tais como convulsões e arritmias } \\
\text { cardíacas. }\end{array}$ \\
\hline 14 & $\begin{array}{c}\text { ORNILLO C e } \\
\text { HARBORD N } \\
\quad(2019)\end{array}$ & $\begin{array}{l}\text { Advances Chronic } \\
\text { Kidney Disease - } \\
\text { Estados Unidos }\end{array}$ & $\begin{array}{l}\text { O tratamento de envenenamento se dá, primeiro, com o suporte à vida, seguido da avaliação da função } \\
\text { dos órgãos. Deve-se considerar o envolvimento de mais de uma substância, com possíveis interações } \\
\text { entre si. Visando evitar a contaminação gastrointestinal, recomenda-se a lavagem gástrica e o carvão } \\
\text { ativado. A alcalinização urinária se faz importante de modo a aumentar a excreção renal. Intoxicações } \\
\text { por medicamentos como bupivacaína, amitriptilina e bupropiona, tem como tratamento favorável a terapia } \\
\text { lipídica intravenosa. A hemodiálise é a opção de tratamento extracorpóreo mais comum. A naloxona foi } \\
\text { reportada como antídoto para intoxicações por opioides. O Diazepam reduz a mortalidade de intoxicação } \\
\text { por cloroquina. Flumazenil é utilizado em overdoses de benzodiazepínicos, apesar de seus efeitos } \\
\text { colaterais. Bicarbonato de sódio para abuso de antidepressivos tricíclicos. A vitamina K foi recomendada } \\
\text { em casos de overdose por varfarina. A acetilcisteína em casos envolvendo paracetamol. }\end{array}$ \\
\hline 15 & $\begin{array}{l}\text { FERRANTI S, et } \\
\text { al. (2018) }\end{array}$ & $\begin{array}{l}\text { Journal of Paediatrics } \\
\text { and Child Health - Itália }\end{array}$ & $\begin{array}{l}\text { Os tratamentos mais comuns para intoxicações por antidepressivos e antiepilépticos são os que evitam } \\
\text { sua digestão, como a lavagem gástrica e o carvão ativado. Estes não são recomendados para } \\
\text { intoxicações por carbamazepina. Estimular a excreção renal a partir da alcalinização da urina é sugerida } \\
\text { em casos de intoxicação por fenobarbitais. Em intoxicações mais severas, deve-se considerar } \\
\text { tratamentos extracorpóreos, como a hemodiálise, a hemoperfusão e a diálise peritoneal. A administração } \\
\text { de flumazenil para tratamento de intoxicações por benzodiazepínicos é controversa, visto que apresenta } \\
\text { efeitos como agitação, convulsões e arritmias. }\end{array}$ \\
\hline 16 & $\begin{array}{l}\text { GHANNOUM M, } \\
\text { et al. (2016) }\end{array}$ & $\begin{array}{l}\text { Clinical Toxicology } \\
\text { (Phila) - Canadá }\end{array}$ & $\begin{array}{l}\text { O uso abusivo de dapsona pode levar à metahemoglobinemia, hemólise e até mesmo à morte. O } \\
\text { tratamento deve visar a descontaminação do trato gastrointestinal com doses múltiplas de carvão ativado, } \\
\text { além da administração de azul de metileno. Para agilizar a eliminação do fármaco, pode-se investir em } \\
\text { tratamentos extracorpóreos, como a hemodiálise com hemoperfusão. }\end{array}$ \\
\hline 17 & $\begin{array}{l}\text { PUTIC V e } \\
\text { JOVIC-STOSIC } \\
\text { J (2015) }\end{array}$ & $\begin{array}{l}\text { Millitary Medical and } \\
\text { Pharmaceutical Journal } \\
\text { of Serbia - Sérvia }\end{array}$ & $\begin{array}{l}\text { As emulsões lipídicas intravenosas, além de serem utilizadas para nutrição de doentes, também têm se } \\
\text { mostrado eficazes para tratamento de intoxicações por fármacos lipofílicos, como anestésicos locais, } \\
\text { bloqueadores de canais de cálcio, digoxina, antipsicóticos, beta bloqueadores e antidepressivos } \\
\text { tricíclicos. }\end{array}$ \\
\hline
\end{tabular}

Fonte: Silva VT, et al., 2021. 
Dentre os 17 estudos selecionados como amostra, 4 trataram sobre a intoxicação por paracetamol (acetaminofeno) (GARZÓN RGC, et al., 2018; CHIEW AL, et al., 2020; MARKS DJB, et al., 2016; STINE JG e LEWIS JH, 2016). O restante abordou sobre medicamentos como antidepressivos, ansiolíticos e antiepilépticos, lítio, ácido acetilsalicílico, barbitúricos, carbamazepina, betabloqueadores, isoniazida, benzodiazepínicos, dapsona e antidepressivos tricíclicos. O suporte básico à vida foi o tratamento de primeira escolha citado pela maioria dos artigos (CASTILHO MM, et al.,2018; SEPULVEDA RA, et al., 2018; PÉREZ MM, et al., 2019; TUÑÓN JGP, et al., 2020; MARTINS LS e FILHO AA, 2018; DIAZ M, et al., 2015; BARTLETT D, 2017; ORNILLO C e HARBORD N, 2019).

Já a administração de antídotos foi descrita por 9 autores, sendo a acetilcisteína, o flumazenil e a naloxona os mais citados, os quais foram prescritos, respectivamente, em intoxicações por paracetamol, benzodiazepínicos e opioides (GARZÓN RGC, et al., 2018; SKOLNIK A e MONAS J, 2017; MARKS DJB, et al., 2016 DIAZ M, et al., 2015; PERNNIGA E, et al., 2015; ORNILLO C e HARBORD N, 2019; CHIEW AL, et al., 2020; STINE JG e LEWIS JH, 2016; FERRANTI S, et al., 2018).

Tratamentos relacionados à adsorção do fármaco também foram descritos, tais como o uso do carvão ativado ou a lavagem gástrica, no entanto, sua aplicação é limitada, visto que depende do tempo de ingestão do medicamento (CASTILHO MM, et al., 2018; SEPULVEDA RA, et al., 2018; PÉREZ MM, et al., 2019; CHIEW AL, et al., 2020; TUÑÓN JGP, et al., 2020; MARTINS LS e FILHO AA, 2018; MARKS DJB, et al., 2016; DIAZ M, et al., 2015; STINE JG e LEWIS JH, 2016; FERRANTI S, et al., 2018; GHANNOUM M, et al., 2016; ORNILLO C e HARBORD N, 2019).

A alcalinização da urina foi sugerida para aumentar a excreção renal do fármaco por 4 autores, sendo 0 bicarbonato de sódio o composto mais utilizado (SEPULVEDA RA, et al., 2018; PÉREZ MM, et al., 2019; FERRANTI S, et al., 2018; ORNILLO C e HARBORD N, 2019). Em casos mais graves, recomendou-se tratamentos extracorpóreos para a purificação do sangue, como a hemodiálise, a diálise peritoneal e a hemoperfusão (CASTILHO MM, et al. (2018; SEPULVEDA RA, et al., 2018; PÉREZ MM, et al., 2019; TUÑÓN JGP, et al., 2020; TUÑÓN JGP, et al., 2020; MARTINS LS e FILHO AA, 2018; ORNILLO C e HARBORD N, 2019; FERRANTI S, et al, 2018; PUTIC V e JOVIC-STOSIC J, 2015).

Também foram encontrados 4 estudos com relatos de terapias alternativas e experimentais usadas para o tratamento de intoxicações por drogas lipofílicas, feitas com emulsificações lipídicas intravenosas, que são utilizadas geralmente para nutrição de paciente. As principais intoxicações por fármacos lipofílicos são por bloqueadores de canais de cálcio, digoxina, antipsicóticos, antidepressivos tricíclicos e betabloqueadores (MARTINS LS e FILHO AA, 2018; BARTLETT D, 2017; ORNILLO C e HARBORD N, 2019; PUTIC V e JOVICSTOSIC J, 2015).

\section{DISCUSSÃO}

A intoxicação medicamentosa é um dos maiores desafios da saúde, não só brasileira, mas também mundial, como evidenciada pelos resultados encontrados, visto que os artigos da amostra são de diversos países. Dentre as principais causas, tem-se o autoextermínio, a automedicação desregrada e ingestões acidentais. No Brasil, enquanto as intoxicações agudas são mais comuns em idosos com intenções suicidas, as intoxicações acidentais são mais comuns em crianças, de acordo com o Ministério da Saúde e com a ANVISA. A automedicação, prática comum e desenfreada entre a população brasileira, é mais propensa de ocorrer em jovens e adultos, segundo o SINITOX. Devido a essa cultura de automedicação, o Brasil ocupa o quinto lugar no ranking de países que mais consomem medicamentos, e a prática corresponde a $35 \%$ das vendas de fármacos no país. Além disso, a prática contribui diretamente para o surgimento de superbactérias resistentes a medicamentos pelo uso abusivo de antibióticos (MATHIAS TL, et al., 2019; SERENO VMB, et al., 2020).

Os principais medicamentos envolvidos em intoxicações brasileiras, segundo Mathias TL (2019), são os benzodiazepínicos, anticonvulsivantes, antidepressivos e analgésicos. O conhecimento da substância ingerida é importante para estabelecer o diagnóstico e o respectivo tratamento, pois alguns fármacos 
apresentam antídotos específicos, enquanto outros precisam ser excretados pela urina ou removidos da corrente sanguínea. Quando a substância é desconhecida, é recomendado pelo Manual de Toxicologia Clínica (2017) a observação dos sintomas apresentados pelo paciente, visto que certos medicamentos são capazes de causar síndromes tóxicas, como a serotoninérgica, sedativo-hipnótica, anticolinérgica, extrapiramidal, opioide, colinérgica ou adrenérgica.

Antes mesmo de descobrir qual o medicamento envolvido na intoxicação, é de extrema importância um exame físico inicial, de modo a estabilizar o paciente. Deve-se atentar às vias aéreas e à respiração, à circulação sanguínea e ao déficit neurológico. A checagem dos sinais vitais, da pressão arterial, dos batimentos cardíacos, da frequência respiratória, das pupilas, da temperatura e da oxigenação se fazem essenciais nesse momento. É indicada a aplicação de glicose e de tiamina (vitamina B1) intravenosa em pacientes inconscientes, visando evitar um quadro de hipoglicemia e um possível rebaixamento de consciência (PÉREZ MM, et al., 2019; CASTILHO MM, et al., 2018; SÃO PAULO, 2017).

Após a estabilização do paciente e a observação de seus sinais clínicos, procura-se o melhor tratamento, considerando seus antecedentes pessoais. Se o medicamento tiver sido ingerido em um determinado intervalo de tempo, entre 2 e 4 horas, pode-se recorrer à lavagem gástrica ou à administração de carvão ativado, substâncias que vão evitar a absorção do medicamento pelo trato gastrointestinal. No entanto, não há consenso médico quanto à utilização do carvão ativado e da lavagem gástrica para o tratamento de algumas intoxicações, como as causadas pela carbamazepina e pela fenitoína. Já em casos de intoxicação por paracetamol, o carvão ativado aparenta reduzir os efeitos hepatotóxicos, além de prevenir a metahemoglobinemia causada pela sobredosagem de dapsona (BEZERRA AAP, et al., 2020; TUÑóN JGP, et al., 2020; STINE JG e LEWIS JH, 2016; FERRANTI S, et al., 2018; GHANNOUM M, et al., 2016).

A alcalinização da urina é indicada para intoxicação por fármacos cuja depuração renal aumenta com o aumento do $\mathrm{pH}$ urinário, como as sobredosagens de ácido acetilsalicílico e de barbitúricos, desde que o paciente não apresente insuficiência renal, pois essa condição limita a excreção do fármaco pela urina. $O$ principal composto para basificar a urina é o bicarbonato de sódio intravenoso (FERRANTI S, et al., 2018; SEPULVEDA RA, et al., 2018). Quando o aumento da excreção renal não se faz suficiente, a hemodiálise, a diálise peritoneal ou a hemoperfusão podem ser solicitadas, visando desintoxicar o sangue do fármaco, sendo utilizada em intoxicações por fenobarbital, lítio e salicilatos (SÃO PAULO, 2017; CASTILHO MM, et al., 2018; SEPULVEDA RA, et al., 2018; PÉREZ MM, et al., 2019).

Em casos de intoxicações por compostos lipofílicos, como antipsicóticos, betabloqueadores e antidepressivos tricíclicos, pode-se utilizar emulsões lipídicas intravenosas, sendo terapias experimentais que apresentam uma alternativa em casos de instabilidade hemodinâmica. A teoria mais aceita para explicar o mecanismo de ação dessas emulsões é que, assim que elas chegam à corrente sanguínea, há a formação de um compartimento que atrai essas substâncias lipofílicas, afastando-as dos receptores teciduais. Além disso, o bicarbonato de sódio pode ser administrado na forma intravenosa, visando desbloquear os canais de sódio afetados pelo excesso de antipsicóticos e antidepressivos tricíclicos (MARTINS LS e FILHO AA, 2018; PUTIC V e JOVIC-STOSIC J, 2015; SKOLNIK A e MONAS J, 2017).

Quando a substância é conhecida, pode-se administrar seu respectivo antídoto, o qual neutralizará a toxicidade do fármaco. É importante enfatizar que nem todas as intoxicações apresentam antídoto, sendo assim, a melhor medida é priorizar o suporte vital básico, tratando os sintomas e evitando possíveis sequelas. A acetilcisteína atua para a redução dos níveis de NAPQI, metabólito tóxico ao fígado do paracetamol, prevenindo uma falência hepática. Pode ser associada ou não à metionina, visto que este fármaco, apesar de contribuir para a redução da hepatoxicidade, também causa efeitos colaterais como vômitos. O flumazenil geralmente é administrado em intoxicações por benzodiazepínicos, pois bloqueia os receptores do composto no sistema nervoso central, impedindo a entrada de íons cloreto no neurônio e a hiperpolarização neuronal. No entanto, há contraindicações, visto que apresenta efeitos colaterais como agitação, arritmias cardíacas e até mesmo convulsões. Quando o agente é algum opioide, o tratamento é feito com naloxona. A vitamina B6 (piridoxina) é capaz de reduzir quadros convulsivos causados pela intoxicação por isoniazida, fármaco utilizado para o tratamento da tuberculose (SÃO PAULO, 2017; GARZÓN RGC, et al., 2018; MARKS DJB, et 
al., 2016; FERRANTI S, et al., 2018; PERNNIGA E, et al., 2015; SKOLNIK A e MONAS J., 2017; ORNILLO C e HARBORD N, 2019).

Com base nas síndromes tóxicas apresentadas pelo paciente, é possível identificar o agente tóxico em questão, sendo possível, então, o tratamento adequado. A síndrome serotoninérgica pode ser causada por intoxicações de fármacos inibidores seletivos de serotonina ou inibidores seletivos de norepinefrina, como o fentanil, os antidepressivos tricíclicos e o tramadol. Entre os achados clínicos de tal síndrome, encontra-se a midríase, agitação, alucinações e convulsões. Nesses casos, pode-se utilizar a ciproheptadina, um antihistamínico antagonista aos receptores serotoninérgicos, além de benzodiazepínicos, visando diminuir os batimentos cardíacos, a agitação e a pressão arterial. Em casos de depressão do sistema respiratório e do sistema nervoso central, acompanhados de miose e bradicardia, suspeita-se de uma síndrome hipnótica sedativa, a qual é causada geralmente por barbitúricos, benzodiazepínicos e opioides. (SÃO PAULO, 2017; BARTLETT D, 2017; PERNNIGA E, et al., 2015)

O presente estudo apresenta limitação quanto às classes dos medicamentos, visto que nem todas estão representadas, como por exemplo os antibióticos, assim como seus respectivos tratamentos e antídotos. Além disso, deve-se enfatizar que o tratamento pode variar de paciente para paciente, visto que a história clínica e antecedentes pessoais influenciam na tomada de decisões terapêuticas. Buscou-se a reunião de diversas intoxicações e seus respectivos tratamentos e antídotos, de forma a contribuir para o meio científico e para a sociedade.

\section{CONSIDERAÇÕES FINAIS}

As intoxicações medicamentosas configuram uma importante problemática, por isso é essencial a divulgação dos tratamentos e dos antídotos disponíveis para cada tipo de intoxicação, de modo a agilizar e aprimorar o atendimento ao paciente. Além disso, são necessários estudos que visem o aperfeiçoamento dos tratamentos já existentes, de modo que a perda de vidas seja mínima. Políticas mundiais e nacionais de prevenção e de educação podem contribuir para reduzir os índices da automedicação e suas possíveis consequências. Tal estudo se faz importante pois reúne os principais medicamentos envolvidos em intoxicações, seus principais tratamentos e antídotos. Sugere-se para estudos futuros a pesquisa de novos tratamentos com terapias alternativas, além de aprimorar as que já existem, tais como as terapias com vitaminas, enzimas e emulsões lipídicas, de modo a abranger o maior número de intoxicações possíveis.

\section{REFERÊNCIAS}

1. ANVISA, Agência Nacional de Vigilância Sanitária. Boletim de farmacoepidemiologia. Disponível em: http://antigo.anvisa.gov.br/resultado-de

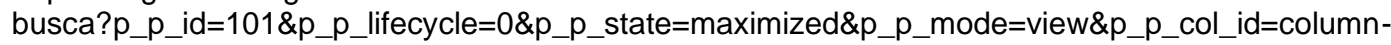

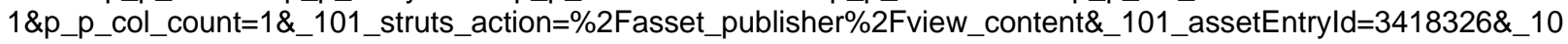
1_type=document. Acessado em: 08 de fevereiro de 2021.

2. BARTLETT D. Drug-Induce serotonin syndrome. Critical Care Nurse, 2017; 37(1): 49-54.

3. BEZERRA ACP, et al. Utilização da lavagem gástrica e do carvão ativado como medidas de intervenção terapêutica na intoxicação exógena. Revista Eletrônica Acervo Saúde, 2020; 12(12): e4990.

4. BRASIL. Ministério da Saúde. Departamento de Informática do SUS. Informações de saúde. Epidemiológicas e morbidade. Intoxicação exógena. Disponível em: http://www2.datasus.gov.br/DATASUS/index. php?area=0203\&id=29878153. Acessado em: 08 de fevereiro de 2021.

5. CARDOSO V, et al. Revisão sistemática de métodos mistos: método de pesquisa para a incorporação de evidências na enfermagem. Texto \& Contexto - Enfermagem, 2019; 28: e20170279.

6. CASTILHO MM, et al. A true dialytic urgency: lithium intoxication. Revista Colombiana de Nefrología, 2018; 5(2): 190194.

7. CHIEW AL, et al. Uptade guidelines for the management of paracetamol poisoning in Australia and New Zealand. The Medical Journal of Australia, 2019; 22(4): 175-183.

8. DÍAZ M, et al. Convulsiones por isoniazida: una causa toxicológica a considerar. Acta toxicológica argentina, 2015; 23(2): 79-82.

9. FERRANTI S, et al. Antiepileptic drugs: Role in paediatric poisoning. Journal of Paediatrics and Child Health, 2018; 54(5), 475-479. 
10. GARZÓN RGC, et al. Actualización en la comprensión y manejo de la intoxicación por acetaminofén. Universitas Medica, 2018; 59(4): 43-53.

11. GHANNOUM M et al. Extracorporeal treatments in a dapsone overdose: a case report. Clinical Toxicology, 2016; 54(9): 886-889.

12. GONÇALVES CA, et al. Intoxicação medicamentosa: relacionada ao uso indiscriminado de medicamentos. Revista Científica da Faculdade de Educação e Meio Ambiente, 2017; 1: 135-143.

13. MARKS DJB, et al. Outcomes from massive paracetamol oversode: a retrospective observational study. British Journal of Clinical Pharmacology, 2016; 83(6): 1263-1272.

14. MARTINS LS e FILHO AA. Uso de solução lipídica em intoxicação por beta-bloqueador: relato de caso. Revista Médica de Minas Gerais, 2017; 28: e1978.

15. MATHIAS TL, et al. Tendências de eventos toxicológicos relacionados a medicamentos atendidos por um Centro de Informações Toxicológicas. Revista brasileira de epidemiologia, 2019; 22: e190018.

16. NÓBREGA HOS, et al. Intoxicações por medicamentos: uma revisão sistemática com abordagem nas síndromes tóxicas. Revista saúde e ciência, 2015; 4 (2): 109-119.

17. OLIVEIRA JFM, et al. Tendência da mortalidade por intoxicação medicamentosa entre gêneros e faixas etárias no Estado de São Paulo, Brasil, 1996-2012. Ciência \& Saúde Coletiva, 2017; 22 (10): 3381-3391.

18. ORNILLO C e HARBORD N. Fundaments of Toxicology-Approach to the Poisoned Patient. Advances in Chronic Kidney Disease, 2020; 27(1): 5-10.

19. PENNINGA E, et al. Adverse Events Associated with Flumazenil Treatment for the Management of Suspected Benzodiazepine Intoxication - A Systematic Review with Meta-Analyses of Randomised Trials. Basic \& Clinical Pharmacology \& Toxicology, 2016; 118(1): 37-44.

20. PÉREZ MM, et al. Intoxicación por barbitúricos, una mirada toxicológica. Horizonte sanitario, 2019; 18(2): $111-118$.

21. PUTIC V e JOVIC-STOSIC J. Intravenous fat emulsion in clinical practice: Nutrient and antidote. Military Medical and Pharmaceutical Journal of Serbia, 2015; 72(3): 274-279..

22. SÃO PAULO. Secretaria Municipal de Saúde. Manual de Toxicologia Clínica: Orientações para assistência e vigilância das intoxicações $\quad$ agudas. $2017 . \quad$ Disponível http://www.cvs.saude.sp.gov.br/up/MANUAL\%20DE\%20TOXICOLOGIA\%20CL\%C3\%8DNICA\%20\%20COVISA\%202017.pdf. Acessado em 08 de fevereiro de 2021.

23. SEPÚLVEDA RA, et al. Intoxicación por ácido acetilsalicílico, fisiopatología y manejo. Revista médica de Chile, 2018; 146(11): 1309-1316.

24. SERENO VMB, et al. Perfil epidemiológico das intoxicações por medicamentos no Brasil entre os anos de 2013 a 2017. Brazilian Journal of Development, 2020; 6(6): 33892-33903.

25. SINITOX. Sistema Nacional de Informações Tóxico Farmacológicas. Dados de Intoxicação Regionais de 2008 a 2013 , 2016. Disponível em: https://sinitox.icict.fiocruz.br/dados-nacionais. Acessado em 08 de fevereiro de 2021.

26. SKOLNIK A e MONAS J. The Crashing Toxicology. Emerg Med Clin N Am, 2020; 38: 841-856.

27. STINE JG e LEWIS JH. Current and future directions in the treatment and prevention of drug-induced liver injury: a systematic review. Expert. Rev. Gastroenterol. Hepatol, 2016; 10(4): 517-536.

28. THORNE PS. Occupational toxicology. In: KLAASSEN, C. D. Casarett and Doull'stoxicology: the basic science of poisons. 8th ed. New York: McGraw-Hill Education, 2013; 1454.

29. TUÑÓN JGP, et al. Intoxicación por carbamazepina: reporte de un caso tratado con hemodiálisis de alto flujo. Medicina crítica (Colegio Mexicano de Medicina Crítica), 2018; 32(5): 290-294. 